\title{
Infrared Spectroscopy of Matrix-Isolated Polycyclic Aromatic Hydrocarbon Cations. 3. The Polyacenes Anthracene, Tetracene, and Pentacene
}

\author{
D. M. Hudgins ${ }^{\dagger}$ and L. J. Allamandola* \\ NASA Ames Research Center, MS 245-6, Moffett Field, Califormia 94035
}

Received: November 9, 1994; In Final Form: March 14, $1995^{\circ}$

NASA-TM-111940

\begin{abstract}
Gaseous, ionized polycyclic aromatic hydrocarbons (PAHs) are thought to be responsible for a very common family of interstellar infrared emission bands. Unfortunately, very little infrared spectroscopic data are available on ionized PAHs. Here we present the near- and mid-infrared spectra of the polyacene cations anthracene, tetracene, and pentacene. We also report the vibrational frequencies and relative intensities of the pentacene anion. The cation bands corresponding to the $\mathrm{CC}$ modes are typically about 10-20 times more intense than those of the $\mathrm{CH}$ out-of-plane bending vibrations. For the cations the $\mathrm{CC}$ stretching and $\mathrm{CH}$ in-plane bending modes give rise to bands which are an order of magnitude stronger than for the neutral species, and the $\mathrm{CH}$ out-of-plane bends produce bands which are 3-20 times weaker than in the neutral species. This behavior is similar to that found for most other PAH cations. The most intense PAH cation bands fall within the envelopes of the most intense interstellar features. The strongest absorptions in the polyacenes anthracene, tetracene, and pentacene tend to group around $1400 \mathrm{~cm}^{-1}$ (between about 1340 and $1500 \mathrm{~cm}^{-1}$ ) and near $1180 \mathrm{~cm}^{-1}$, regions of only moderate interstellar emission. These very strong polyacene bands tend to fall in gaps in the spectra of the other PAH cations studied to date suggesting that while PAHs with polyacene structures may contribute to specific regions of the interstellar emission spectra, they are not dominant members of the interstellar PAH family.
\end{abstract}

\section{Introduction}

During the past decade evidence has been mounting that polycyclic aromatic hydrocarbons (PAHs) are important components of the interstellar medium.' Under the harsh conditions in the interstellar emission zones, most PAHs are expected to be positively charged. For this reason we have undertaken a study of the infrared spectroscopic properties of matrix-isolated PAH cations. While the primary motivation for this research is to investigate the role of PAHs in the interstellar medium, the results are of general interest. PAH cations are considered to be important intermediates in combustion, ${ }^{2}$ and some PAH intermediates and primary reaction products are carcinogenic. ${ }^{3}$

Ionized ${ }^{4}$ and neutral ${ }^{5}$ PAH molecules have been proposed as the carriers of a very common family of interstellar infrared fluorescence bands. The ubiquity of this spectrum suggests that free molecular PAHs are common throughout interstellar space, and the intensity of the bands indicates that they are as abundant as the most abundant polyatomic interstellar molecules known. As much as $30-40 \%$ of the radiant energy from some of these sources is emitted in this family of bands. Reviews of this field can be found in ref 1 . While the interstellar PAH hypothesis rests on the general resemblance of the interstellar emission spectra to the absorption spectra of aromatic hydrocarbons, the available data are mainly for neutral PAHs suspended in salt pellets ${ }^{6}$ or organic solvents. ${ }^{7}$ Under such conditions interactions with the surrounding media and with other PAH molecules (clusters) strongly perturb the transitions. ${ }^{8}$ While this body of information has been invaluable in establishing the interstellar PAH hypothesis, there are important differences in detail between the laboratory and interstellar spectra. Differences in band position and relative band intensities, as well as the fact that most interstellar PAHs are expected to be ionized in the

* To whom correspondence should be addressed

Current address: Department of Chemistry, Adrian College, Adrian, MI 49221.

\& Abstract published in Advance ACS Abstracts, May 1. 1995. regions where they emit, have hindered proper testing of the hypothesis and the possible exploitation of PAHs as probes of the interstellar medium. Thus, the infrared spectra of neutral, isolated PAHs and of ionized, isolated PAHs are of fundamental importance to further progress in this field.

In response to this need for reliable, quantitative, infrared spectroscopic data on neutral and ionized PAHs under conditions relevant to astrophysical environments, we have initiated a systematic program to obtain this information. There are many PAHs which one can study. The PAHs studied at the NASA Ames Research Center have been selected for the following reasons. First, as little is known about the infrared properties of PAH cations, the smallest members of the PAH family were chosen to minimize the number of fundamental vibrations and spectral complexity. Second, as this work is motivated by the astrophysical question, the PAHs most likely to be important in this context were given preference. Third, as the effects of structure and size on the infrared spectra of simple PAH cations are not known, related PAHs with limited, well-defined structural differences have been studied together. Finally, as size increases, PAH samples tend to become increasingly refractory and increasingly difficult and expensive to obtain. Thus, in the interest of experimental practicality, smaller PAHs were the logical starting point. Small PAHs are also relevant from the astrophysical point of view. Molecules containing 2030 carbon atoms are thought to dominate the interstellar fluorescence at the shortest wavelengths. ${ }^{\text {la. }} 4$

In paper 1 of this series, our experimental approach and the $4000-200 \mathrm{~cm}^{-1}$ spectra of the smallest PAH, naphthalene $\left(\mathrm{C}_{10} \mathrm{H}_{8}\right)$, and its fully deuterated analog $\left(\mathrm{C}_{10} \mathrm{D}_{8}\right)^{9}$ are presented. Paper 2 reports the near- and mid-infrared spectral properties of the thermodynamically most favored PAHs containing between 6 and 24 carbon atoms (phenanthrene, pyrene, benzo[e]pyrene, benzo[ghi]perylene, and coronene). ${ }^{10}$ Here we focus on the properties of several members of the polyacene family. comparing the spectra, whenever possible, with previously 
published data. $9.11,12$ This phase of the program is designed to investigate frequency and intensity trends which may be associated with a specific geometry, in this case a linear chain of hexagonal aromatic moieties of varying length. In subsequent papers in this series other aspects of PAH cation structure will be explored. The neutral spectra of these PAHs will also be presented elsewhere. ${ }^{13.14}$ The goal is to understand how structure and size influence the infrared spectra of small PAHs in their neutral and cationic forms.

Efforts to provide this type of information are now underway at several institutions. Defrees and Miller theoretically predicted surprising relative infrared band strength differences between the neutral and ionized forms of the PAHs naphthalene and anthracene. ${ }^{15 a}$ Subsequently, Defrees et al..$^{15 b}$ and Pauzat et al. ${ }^{16}$ greatly expanded this work. Prof. Vala and co-workers at the University of Florida have published the infrared spectroscopic properties for cations of the matrix isolated PAHs naphthalene, anthracene, pyrene, perylene, and coronene, ${ }^{11.12 .17 .18}$ and d'Hendecourt et al. have reported the spectrum of the coronene cation. ${ }^{19}$

This paper is laid out as follows. The experimental technique is briefly summarized in section II. In section III criteria used to identify the cation bands are described. Section IV presents the near- and mid-infrared spectra of the anthracene and tetracene cations and of the pentacene anion and cation. These results are also discussed in section IV, where the spectra are compared to that of naphthalene and trends are pointed out. Finally, the astrophysical implications are described in section V.

\section{Experimental Section}

Individual PAH molecules are isolated in an argon matrix where they are photoionized and probed spectroscopically. The techniques will be described only briefly as our experimental procedure has been presented in detail previously. 9.10 Matrix samples were deposited on a $10 \mathrm{~K} \mathrm{CSI}$ window suspended in an ultrahigh-vacuum chamber ( $\left.p \sim 10^{-8} \mathrm{mTor \pi}\right)$. This chamber is externally supported and suspended in the sample compartment of a Fourier transform infrared spectrometer. Samples were prepared by codeposition of a gaseous PAH with an overabundance of argon. After sample deposition was complete, the CsI cold window was rotated to face the beam of an infrared spectrometer and a prephotolysis spectrum recorded. The sample was then rotated to face a microwave-powered discharge lamp for photolysis. After exposure to the ionizing radiation, the postphotolysis spectrum was recorded. Comparison of this spectrum with the neutral spectrum permitted identification of the ion features which appeared upon photolysis.

For deposition, PAH samples were placed in resistively heated, $1 / 2$ in. diameter, Pyrex tubes mounted on the sample chamber. Tube temperature was monitored using a chromel alumel thermocouple mounted on the exterior of the tube with aluminum metal tape. Such an arrangement should potentially be useful at temperatures as high as $316^{\circ} \mathrm{C}$ (the failure temperature of the Viton O-ring) and with samples as small as a few milligrams. Argon was admitted through a second port at a position $45^{\circ}$ from the first in such a way that the two "streams" coalesced before the surface of the cold window. The deposition tubes ended between 2 and $4 \mathrm{~cm}$ from the cold window. During deposition this window was maintained at a position intermediate to the two deposition ports. The argon deposition line was liquid nitrogen trapped to minimize contamination. Sample quality was found to be optimal for PAH vapor pressures in the range from 10 to $30 \mathrm{mTorr}$. Higher vapor pressures required higher argon deposition rates which exceeded the thermal conductivity of the CsI window, warming the matrix. The "annealing" which resulted produced a matrix which was highly scattering at short wavelengths, crippling the Ly- $\alpha$ photoionization efficiency and the signal-to-noise ratio of the near-IR spectrum. Conversely, lower vapor pressures required longer deposition times, which necessarily increased the contaminants in the matrix and, in turn, reduced the ionization efficiency. Thermochemical data ${ }^{20}$ were used to establish the approximate temperature necessary for each PAH investigated. Optimum tube temperatures were as follows: anthracene, 33 ${ }^{\circ} \mathrm{C}$; tetracene, $109{ }^{\circ} \mathrm{C}$; and pentacene, $203{ }^{\circ} \mathrm{C}$. The optimal argon flow rate was estimated to be between 0.5 and $1.0 \mathrm{mmol} /$ h.

PAH/Ar matrices were photoionized using the Lyman- $\alpha$ emission from a microwave-powered discharge lamp using a $10 \% \mathrm{H}_{2}$ in $\mathrm{He}$ gas mixture at $\sim 75$ mTorr. $\mathrm{A} \mathrm{MgF}_{2}$ vacuum window allowed transmission of this radiation to the sample. Those experiments which incorporated the electron acceptor $\mathrm{CCl}_{4}$ in the matrix necessitated the lower energy photolysis ${ }^{10}$ generated by a discharge in pure $\mathrm{H}_{2}$ gas at $\sim 150$ mTorr. This optimized the broad molecular hydrogen emission band centered around $160 \mathrm{~nm}(7.77 \mathrm{eV})$ with respect to the $\mathrm{Ly}-\alpha$ line. $\mathrm{A} \mathrm{CaF}_{2}$ vacuum window (cutoff $\lambda \approx 150 \mathrm{~nm}$ ) served as a filter to exclude the residual $L y-\alpha$ radiation. Ion yield upper limits ranging from $5 \%$ to $10 \%$ are typically realized with this technique. The upper limits of the yields for the spectra presented here were as follows: anthracene, $5.5 \%$; tetracene. $11 \%$; and pentacene, $12 \%$.

All spectra reported here were measured at $0.5 \mathrm{~cm}^{-1}$ resolution. This resolution is critical for detecting ion bands which fall near the position of a neutral band. Spectra were typically generated through coaddition of five blocks of 200 scans, a number which optimized both the signal-to-noise ratio and time requirements of each experiment. Mid-infrared spectra (7000-500 $\mathrm{cm}^{-1}$ ) were collected using an MCT-B detector' KBr beam splitter combination. Near-infrared spectra (15 100$8800 \mathrm{~cm}^{-1}$ ) were collected using a silicon detector/quartz beam splitter combination. Conversion between the two spectral regions allowed measurement of both electronic and vibrational band intensities from a single matrix.

Tetracene (Aldrich Chemical Co., $98+\%$ purity), pentacene (Aldrich Chemical Co., 98+\% purity), and argon (Matheson prepurified, $99.998 \%$ minimum) were used without further purification.

\section{Cation Band Identification}

As in previous studies of PAH cation spectra, three criteria must be met before a photoproduct band is attributed to a particular PAH cation. First, the bands must only appear when the associated neutral PAH is present in the matrix. A number of control experiments have been carried out to ensure that this is the case. Second, the bands attributed to the cation must be markedly enhanced when $\mathrm{CCl}_{4}$, an electron acceptor, is present in the matrix at a concentration of 1 part in 200. This behavior establishes that the bands arise from a positive ion. This effect is demonstrated in Figure 1, which shows part of the tetracene cation spectrum generated both with and without $\mathrm{CCl}_{4}$ in the matrix. Third, all the bands attributed to the cation must evolve in a similar fashion. Spectra collected as a function of photolysis time show that all of the bands assigned to the respective cation grow and decline together in a manner distinct from other features produced by photolysis. These band correlation plots are presented below along with the appropriate spectroscopic data. In general, the intensities of the bands corresponding to the PAH cation peak after $4-8 \mathrm{~min}$ of photolysis and then 


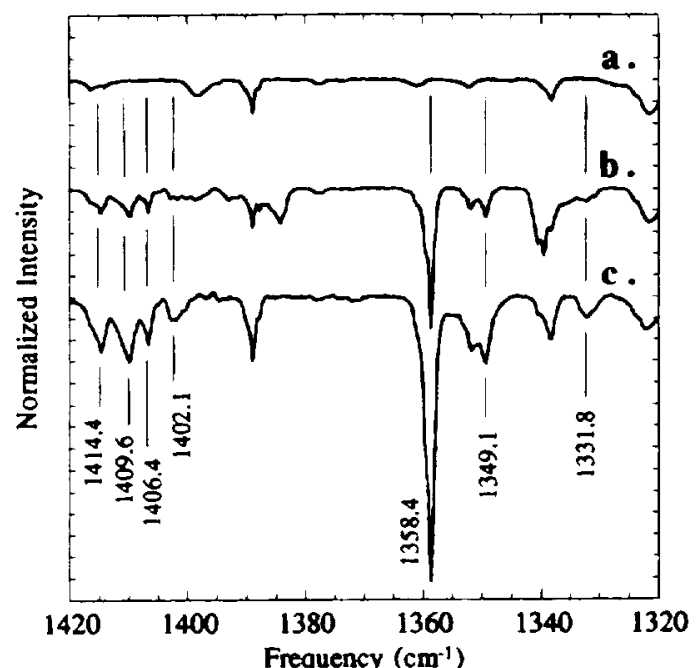

Figure 1. Enhancement of the tetracene cation yield with the addition of $\mathrm{CCl}_{4}$ to the matrix. The $1420-1320 \mathrm{~cm}^{-1}$ region of the spectrum of matrix-isolated tetracene (a) before photolysis, (b) after $8 \mathrm{~min}$ of insitu photolysis. and (c) after $8 \mathrm{~min}$ of in-situ photolysis of a matrix containing $\mathrm{CCl}_{4}$ at the level of 1 part in 200 . Tetracene cation bands are labeled.

remain essentially constant or fall off slightly upon further photolysis. This behavior is similar to that found for the other PAH cations. ${ }^{9} .10$ Conversely the bands of other photoproducts tend to increase steadily with photolysis time. Other photoproduct bands were not common, typically numbering $2-4$ in each case. Most common were the $904 \mathrm{~cm}^{-1} \mathrm{HAr}_{2}{ }^{+}$and 1589 $\mathrm{cm}^{-1}$ bands. These appeared moderately strong in a number of experiments. Other weak photoproduct bands at 1388 and $1104 \mathrm{~cm}^{-1}\left(\mathrm{HO}_{2}{ }^{+}\right)$and at $1039 \mathrm{~cm}^{-1}\left(\mathrm{O}_{3}\right)$ appeared in several experiments, as did bands attributable to $\mathrm{CO}_{2}$ and possibly $\mathrm{CO}$.

\section{Results}

The positions and relative intensities of the mid-infrared cation bands for the polyacenes anthracene $\left(\mathrm{C}_{14} \mathrm{H}_{10}{ }^{+}\right)$, tetracene $\left(\mathrm{C}_{18} \mathrm{H}_{12}{ }^{+}\right)$, and pentacene $\left(\mathrm{C}_{22} \mathrm{H}_{14}{ }^{+}\right)$are compared with those of naphthalene $\left(\mathrm{C}_{10} \mathrm{H}_{8}{ }^{+}\right)$in Table 1 . These spectra are discussed below. Only those regions of the spectrum where cation bands appear are shown. The spectrum of the pentacene anion is also discussed here. The complete spectrum of the neutral isolated PAHs will be given in ref 13 . The spectral properties of the naphthalene and anthracene cations, the first two members of the series, have also been presented elsewhere. ${ }^{9 .} 11.12$

A. Mid-Infrared Spectroscopy. As is the case with previous PAH cations studied, no new features were found in the $\mathrm{CH}$ stretching region between 3200 and $2900 \mathrm{~cm}^{-1}$ for the cations presented here. Theoretical calculations have predicted that these modes are suppressed in the cations. ${ }^{15 a . b .16}$ The continuing failure to observe any $\mathrm{CH}$ stretching features whatsoever attributable to a cation is certainly consistent with this prediction. It is likely that the presumably weaker cation bands are screened by the corresponding bands of the more abundant neutral parent.

The Anthracene Cation, $\mathrm{C}_{14} \mathrm{H}_{10}{ }^{+}$. The mid-infrared spectrum of the anthracene cation is shown in Figure 2. The band frequencies and relative intensities are listed in Table 1 . The evolution of the bands assigned to the cation with $L y-\alpha$ photolysis is plotted in Figure 3 . The bands reported here compare favorably with those reported previously by Szczepanski et al. ${ }^{12}$ We have detected additional bands attributable to the anthracene cation. They lie at 748.3, 1183.3, 1314.6, 1352.6, 1364.4. 1406.1, 1430.2, and $1586.4 \mathrm{~cm}^{-1}$. Szczepanski et al.
TABLE 1: Infrared Frequencies and Relative Intensities for the Polyacene Cations through Pentacene

\begin{tabular}{|c|c|c|c|c|c|c|c|}
\hline \multicolumn{2}{|c|}{ naphthalene } & \multicolumn{2}{|c|}{ anthracene } & \multicolumn{2}{|c|}{ tetracene } & \multicolumn{2}{|c|}{ pentacene } \\
\hline$v\left(\mathrm{~cm}^{-1}\right)$ & $I_{\mathrm{rel}}$ & $v\left(\mathrm{~cm}^{-1}\right)$ & $I_{\text {rel }}$ & $v\left(\mathrm{~cm}^{-1}\right)$ & $I_{\text {rel }}$ & $v\left(\mathrm{~cm}^{-1}\right)$ & $I_{\mathrm{rel}}$ \\
\hline 758.7 & 0.27 & 748.3 & 0.263 & 929.4 & 0.0459 & 740.8 & 0.00304 \\
\hline 1023.2 & 0.054 & 912.0 & 0.0930 & 1155.8 & 0.0116 & 748.5 & 0.0536 \\
\hline 1214.9 & 0.2 & 1183.3 & 0.0142 & 1178.5 & 0.437 & 862.0 & 0.0159 \\
\hline 1218.0 & 1.0 & 1188.6 & 0.700 & 1302.9 & 0.00943 & 933.9 & 0.0613 \\
\hline 1400.9 & 0.044 & 1290.4 & 0.0580 & 1331.8 & 0.0575 & 1149.9 & 0.0327 \\
\hline 1518.8 & 0.1 & 1314.6 & 0.0595 & 1349.1 & 0.328 & 1171.6 & 0.00284 \\
\hline 1525.7 & 0.29 & 1341.0 & 1.00 & 1358.4 & 1.00 & 1174.7 & 0.415 \\
\hline & & 1352.6 & 0.307 & 1371.2 & 0.0132 & 1232.8 & 0.313 \\
\hline & & 1364.4 & 0.0383 & 1402.1 & 0.0384 & 1306.0 & 0.0378 \\
\hline & & 1406.1 & 0.0149 & 1406.4 & 0.0690 & 1339.3 & 0.0392 \\
\hline & & 1409.5 & 0.105 & 1409.6 & 0.180 & 1361.7 & 0.321 \\
\hline & & 1418.4 & 0.861 & 1414.4 & 0.0888 & 1365.6 & 0.141 \\
\hline & & 1430.2 & 0.0143 & 1422.5 & 0.0270 & 1384.0 & 0.0398 \\
\hline & & 1456.5 & 0.0733 & 1478.3 & 0.531 & 1395.5 & 1.00 \\
\hline & & 1539.9 & 0.152 & 1488.0 & 0.129 & 1399.0 & 0.00582 \\
\hline & & 1586.4 & 0.138 & 1543.3 & 0.229 & 1417.8 & 0.331 \\
\hline & & & & 1604.7 & 0.0172 & 1436.0 & 0.00754 \\
\hline & & & & & & 1448.8 & 0.0199 \\
\hline & & & & & & 1487.5 & 0.266 \\
\hline & & & & & & 1495.5 & 0.0571 \\
\hline & & & & & & 1504.7 & 0.0895 \\
\hline & & & & & & 1531.1 & 0.00684 \\
\hline & & & & & & 1534.3 & 0.00661 \\
\hline
\end{tabular}

report bands at 1034 and $432 \mathrm{~cm}^{-1}$, which we did not detect. While the $432 \mathrm{~cm}^{-1}$ feature was out of the range of our instrument, that at $1034 \mathrm{~cm}^{-1}$ was reported as moderately strong and should have been detected as the neutral molecule does not possess absorptions which screen this area. We note that we have observed an $\mathrm{O}_{3}$ impurity band in several of our experiments close to this frequency.

The mid-infrared spectrum of the anthracene cation is dominated by the three strong bands at 1418.4, 1341, and 1188.6 $\mathrm{cm}^{-1}$. The first has been assigned to a $\mathrm{CC}$ stretch, while the latter two have been attributed to in-plane $\mathrm{CH}$ bends by Szczepanski et al. ${ }^{12}$ With an intensity of 0.70 relative to that of the most intense band in the cation spectrum, the 1188.6 $\mathrm{cm}^{-1}$ band is particularly notable. The cations of the thermodynamically most favored PAHs show, if anything, only very weak ion features in this region (relative intensity typically $\leq 0.03$ ). Such a marked difference is indicative that cation structure has a strong influence on the intensity of the $\mathrm{CH}$ inplane bending vibration.

The bands at 912.0 and $748.3 \mathrm{~cm}^{-1}$ are likely due to $\mathrm{CH}$ out-of-plane bends. These correspond nicely with the $\mathrm{CH}$ outof-plane bending frequencies predicted by Szczepanski et al. at 920 and $748 \mathrm{~cm}^{-1}$. Anthracene has two rings with four adjacent $\mathrm{H}$ atoms and one with two nonadjacent $\mathrm{H}$ atoms. The 912.0 $\mathrm{cm}^{-1}$ band falls just beyond the high frequency limit of the normal range expected for nonadjacent $H$ atoms, and the 748.3 $\mathrm{cm}^{-1}$ band falls right in the range expected for neutral PAHs with four adjacent $\mathrm{H}$ atoms on a ring.

By an analysis similar to that set forth previously for the cations of the thermodynamically most favored series of PAHs, ${ }^{10}$ we can estimate the intensity of the $\mathrm{CH}$ out-of-plane bending modes in the anthracene cation relative to those of the neutral molecule. By direct measurement of the attenuation of the bands of neutral anthracene upon photolysis, we calculate an upper limit to the percent ionization $\left(\left(N^{+} \mathrm{N}^{0}\right) \times 100\right)$ of $5.5 \%$. Therefore, if the $\mathrm{CH}$ out-of-plane bending modes in the anthracene cation were equal in intensity to those in the neutral molecule, we would expect these bands to appear in the spectrum with an intensity $5.5 \%$ that of the prephotolysis neutral bands. In fact, we find that the intensities of the 912.0 and 

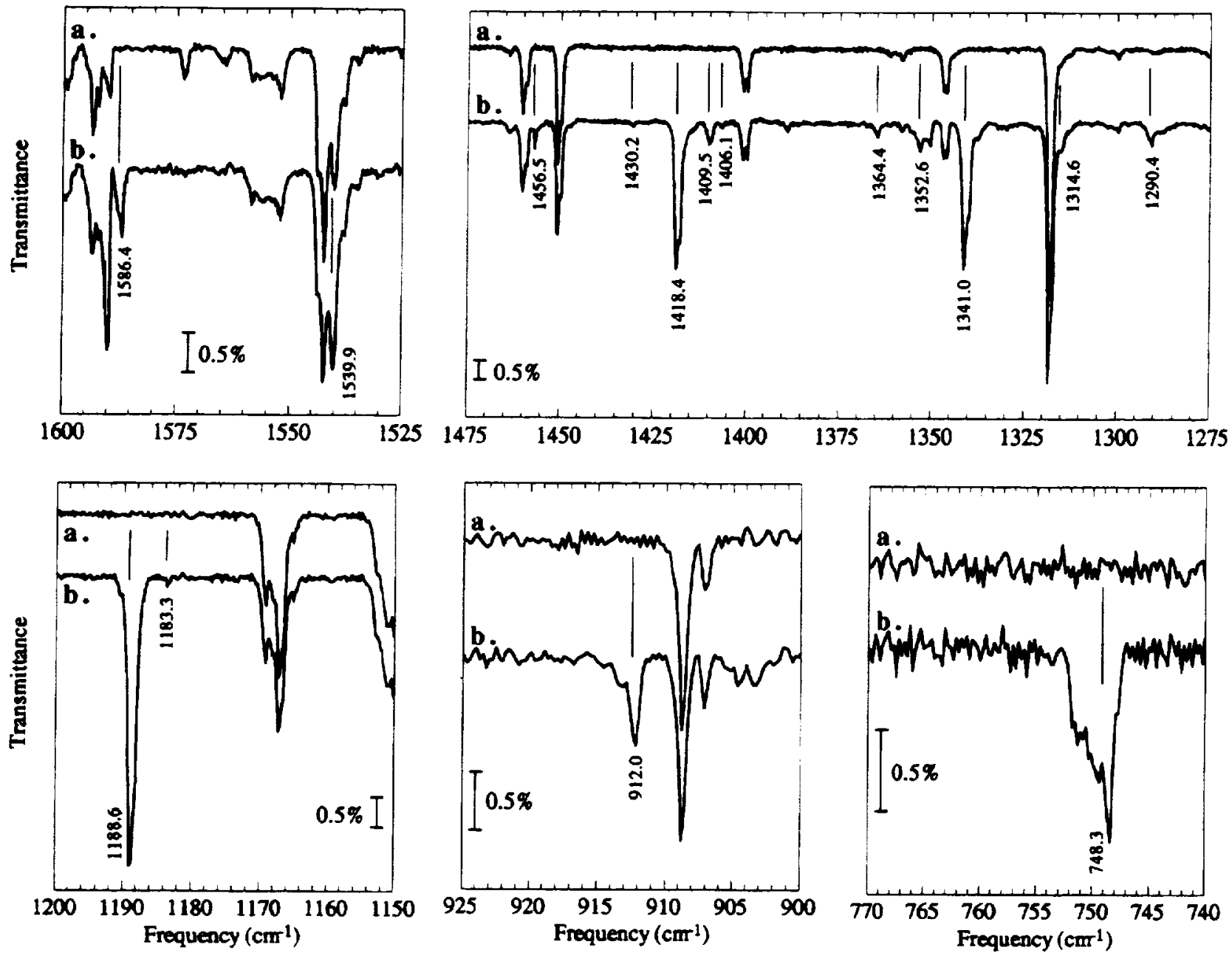

Figure 2. Mid-infrared spectrum of the anthracene cation isolated in an argon matrix at $10 \mathrm{~K}$ : (a) before photolysis; (b) after 8 min in-situ photolysis. The cation bands are labeled with their positions.

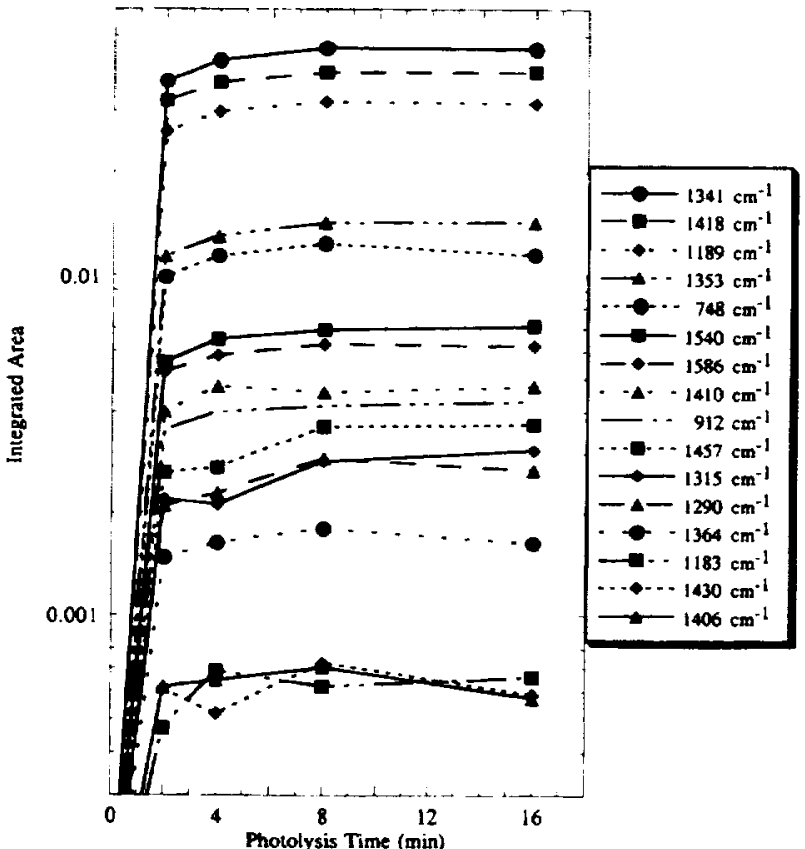

Figure 3. Growth of the anthracene cation integrated photoproduct band areas $\left(\mathrm{cm}^{-1}\right)$ as a function of photolysis time.

$748.3 \mathrm{~cm}^{-1} \mathrm{CH}$ out-of-plane cation bands are reduced from this projected value by factors of 5.5 and 3 , respectively.
The Tetracene Cation, $\mathrm{C}_{18} \mathrm{H}_{12}{ }^{+}$. The mid-infrared spectrum of the tetracene cation is shown in Figure 4. The band frequencies and relative intensities are listed in Table 1 . The evolution of the bands assigned to the cation with $L y-\alpha$ photolysis is plotted in Figure 5. As with several ions previously studied, this band correlation, one of the criteria of ion assignment, is looser for the weaker bands whose signal-tonoise ratios lie in the $2-3$ range. Nonetheless, their overall behavior of a sharp rise and leveling off warrants their assignment to the cation.

Most of the bands attributed to the tetracene ion fall between about 1600 and $1100 \mathrm{~cm}^{-1}$. Thus, they are assigned principally to $\mathrm{CC}$ stretching and $\mathrm{CH}$ in-plane bending modes. Again, notable is the presence of an intense feature in the vicinity if $1180 \mathrm{~cm}^{-1}$. This band, falling at $1178.5 \mathrm{~cm}^{-1}$, has a relative intensity of $\sim 0.44$. The presence of such a feature in both the anthracene and tetracene cation spectra indicates that the linear geometry of the molecules favors the intensity of this vibrational mode. There are undoubtedly other cation bands which are screened by neutral features. Due to unavoidable confusion from $\mathrm{H}_{2} \mathrm{O}$ contamination in the matrix, it is difficult to ascertain whether or not all of the tetracene cation bands which fall in the $1600 \mathrm{~cm}^{-1}$ region have been detected. Only one new band $\left(929 \mathrm{~cm}^{-1}\right.$ ) falls close to the region associated with $\mathrm{CH}$ outof-plane bending vibrations (about 900 and $600 \mathrm{~cm}^{-1}$ ).

The cation frequency spectrum, with most bands between 1600 and $1200 \mathrm{~cm}^{-1}$, does not resemble that of the neutral species which has bands evenly distributed between 1700 and 

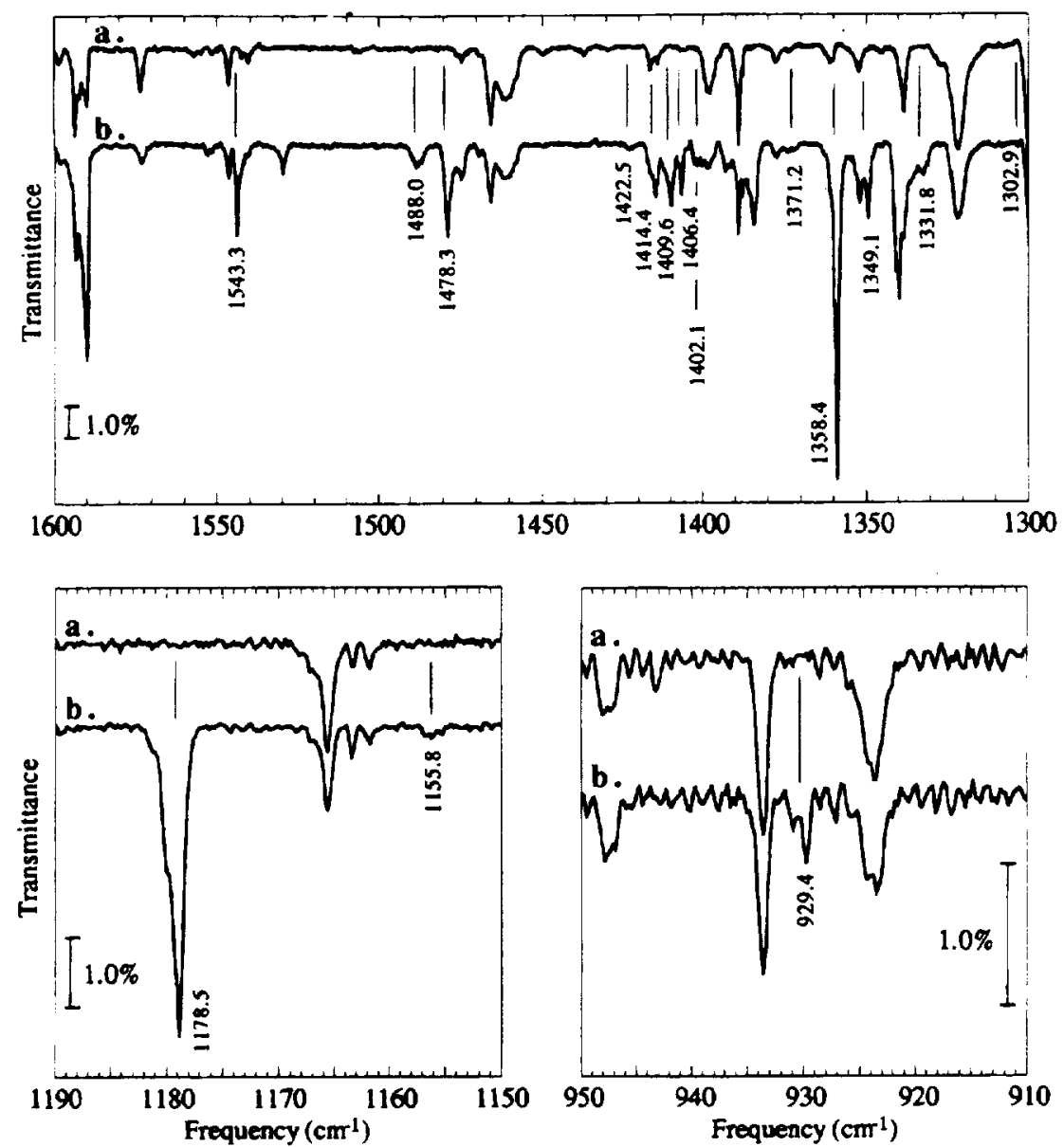

Figure 4. Mid-infrared spectrum of the tetracene cation isolated in an argon matrix at $10 \mathrm{~K}$ : (a) before photolysis; (b) after 8 min in-situ photolysis. The cation bands are labeled with their positions. The band at $1604.7 \mathrm{~cm}^{-1}$ is not shown.

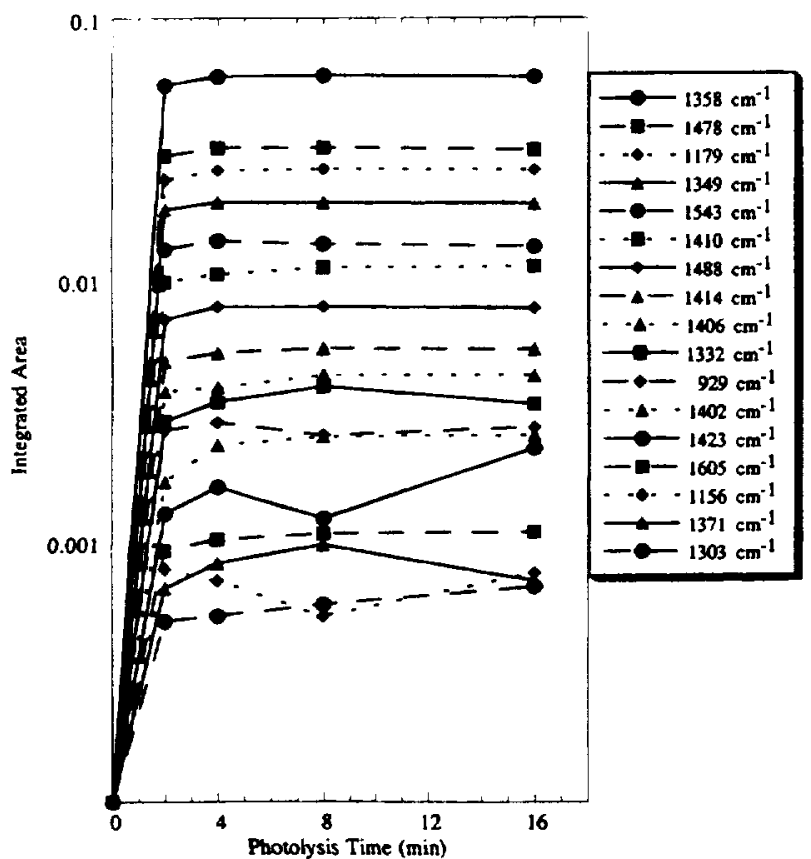

Figure 5. Growth of the tetracene cation integrated photoproduct band areas $\left(\mathrm{cm}^{-1}\right)$ as a function of photolysis time.

$700 \mathrm{~cm}^{-1}$. The intensity pattern is also remarkably different, a characteristic for all PAH cations studied to date. While neutral tetracene has weak to moderate bands between 1700 and 900 $\mathrm{cm}^{-1}$ (the $\mathrm{CC}$ stretching and $\mathrm{CH}$ in-plane bending regions) and two very strong bands at about 895 and $740 \mathrm{~cm}^{-1}$ (out-of-plane
$\mathrm{CH}$ bends), the cation band intensity pattern is just the opposite, with the bands in the $\mathrm{CC}$ stretching and $\mathrm{CH}$ out-of-plane bending region dominating. Interestingly, the strongest cation bands are at 1478 and $1358 \mathrm{~cm}^{-1}$, frequencies that lie between the strongest interstellar emission features which fall at about 1613 and $1300 \mathrm{~cm}^{-1}$. Other strong tetracene cation bands are at 1543.3 and $1178.5 \mathrm{~cm}^{-1}$.

Only one cation band, that at $929.4 \mathrm{~cm}^{-1}$, has been detected which may be associated with a $\mathrm{CH}$ out-of-plane bending mode of the tetracene cation. This molecule has two rings with four adjacent $\mathrm{H}$ atoms and two with two nonadjacent $\mathrm{H}$ 's. It is reasonable to expect that the band at $929.4 \mathrm{~cm}^{-1}$ arises from the out-of-plane bending vibration of the nonadjacent H's. Similar to the results found for many of the PAH cations previously, the mode is blue-shifted by $\sim 30 \mathrm{~cm}^{-1}$ from its frequency in the neutral molecule $\left(895 \mathrm{~cm}^{-1}\right)$. As such, the band does not fall in the range classically associated with nonadjacent hydrogens, but several $\mathrm{cm}^{-1}$ beyond the highfrequency limit. This is consistent with previous results which have indicated that the force field for the cation is quite different from that of the neutral. Based on the measured upper limit of $11 \%$ ionization and using an argument analogous to that presented for the anthracene cation, the nonadjacent $\mathrm{CH}$ outof-plane mode of the tetracene cation is suppressed by a factor of 11 from its value in the neutral molecule.

The Pentacene Cation, $\mathrm{C}_{22} \mathrm{H}_{14}{ }^{+}$. The mid-infrared absorption spectrum of the pentacene cation is shown in Figure 6 . The cation band frequencies and relative intensities are listed in Table 1. The evolution of the bands assigned to $\mathrm{C}_{20} \mathrm{H}_{12}{ }^{+}$with $\mathrm{Ly}-\alpha$ photolysis is plotted in Figure 7. 

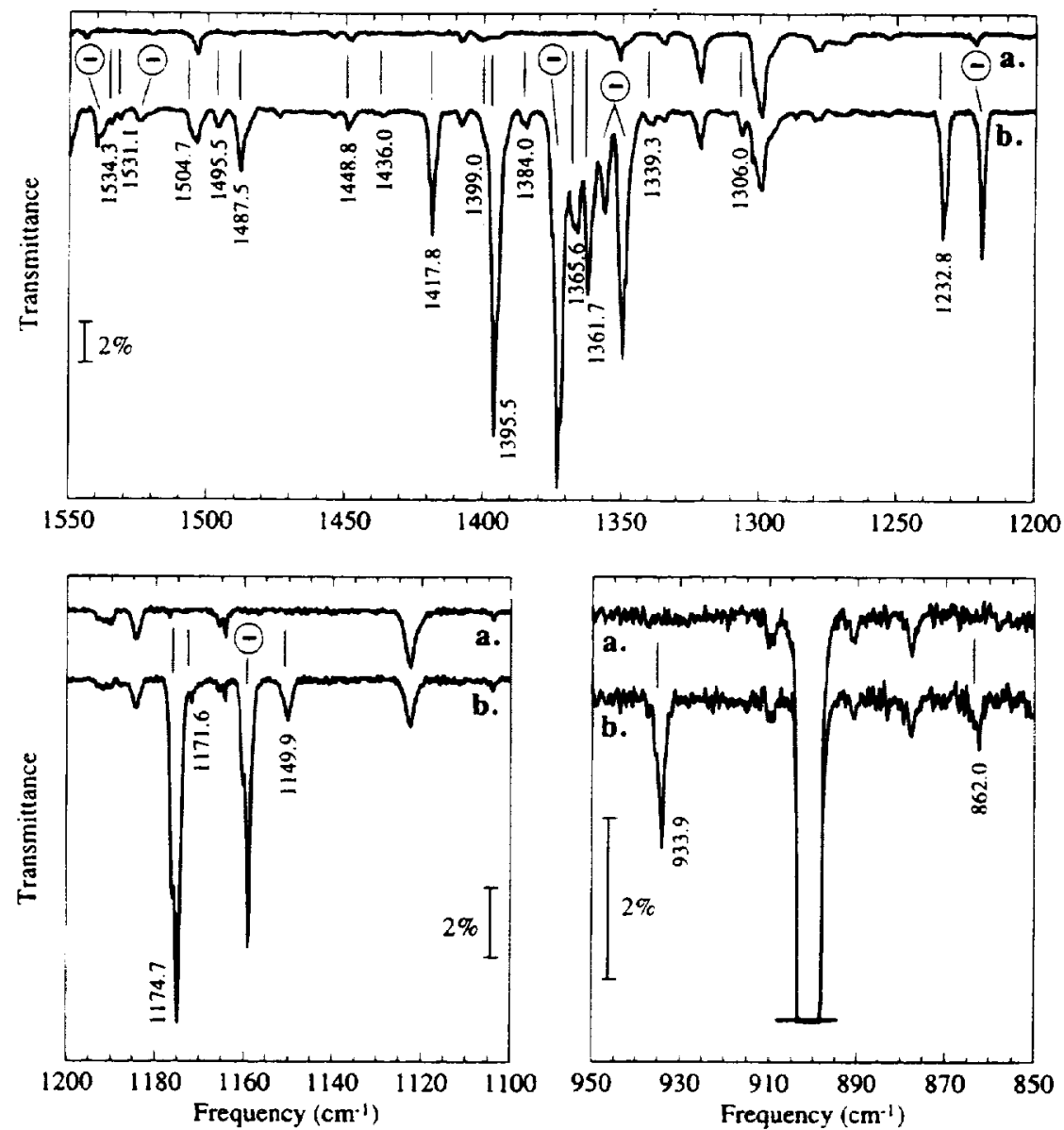

Figure 6. Mid-infrared spectrum of the pentacene cation and anion isolated in an argon matrix at $10 \mathrm{~K}$ : (a) before photolysis: (b) after 8 min in-situ photolysis. The cation bands are labeled with their positions. The anion bands are labeled with a minus sign. Due to its low intensity and separation from other features, the cation bands at 740.8 and $748.5 \mathrm{~cm}^{-1}$ are not shown.

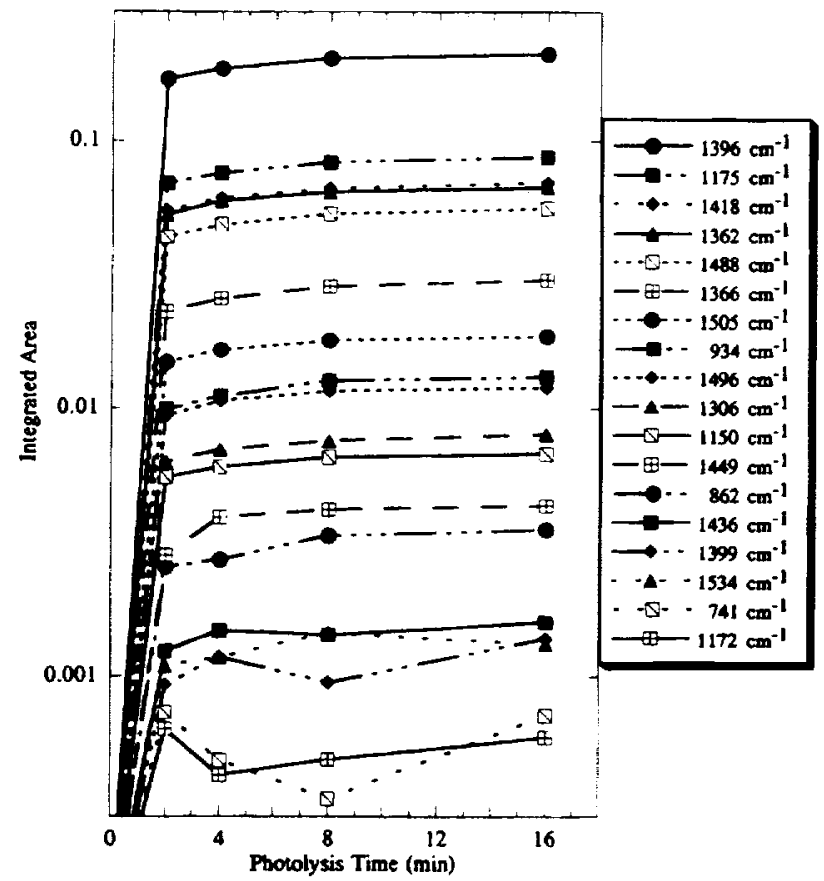

Figure 7. Growth of the pentacene cation integrated photoproduct band areas $\left(\mathrm{cm}^{-1}\right)$ as a function of photolysis time. The behavior of the 748.5 . $1232.8,1339.3,1384.0$, and $1531.7 \mathrm{~cm}^{-1}$ bands is not shown to preserve clarity. They follow the same pattern as given here.

As with tetracene, the cation spectrum does not resemble that of the neutral species at all. The moderately strong cation bands detected all lie between about 1500 and $1170 \mathrm{~cm}^{-1}$, with one strong absorption at $1395.5 \mathrm{~cm}^{-1}$. In contrast, the strongest bands in the neutral molecule occur at about 900 and $732 \mathrm{~cm}^{-1}$. and the moderately intense bands are evenly distributed over a much wider range-between 2000 and $500 \mathrm{~cm}^{-1}$. Both the anthracene and tetracene cations displayed a strong $\mathrm{CH}$ in-plane bending feature near $1180 \mathrm{~cm}^{-1}$. If, as suggested earlier, the polyacene geometry is conducive to strong activity in this mode, such a feature would be expected in the spectrum of the pentacene cation as well. Indeed, the pentacene cation does have a feature falling at $1174.5 \mathrm{~cm}^{-1}$ with a relative intensity of 0.42 .

In addition to the $1174.5 \mathrm{~cm}^{-1}$ band and other weak features detected in the $\mathrm{CH}$ in-plane bending regions, several weak features also appear in the $\mathrm{CH}$ out-of-plane bending region. Pentacene contains two rings with quadruply adjacent $\mathrm{H}$ atoms and three with two nonadjacent hydrogen atoms. The out-ofplane bending features of neutral pentacene appear at 900, 824, and $731 \mathrm{~cm}^{-1}$. The cation exhibits bands at $933.9,862.0$, and $740.8 \mathrm{~cm}^{-1}$. The latter two bands lie in the $\mathrm{CH}$ out-of-plane bending regions classically associated with respectively nonadjacent and quadruply adjacent $\mathrm{H}$ atoms on neutral aromatic hydrocarbons. On the other hand, the $933.9 \mathrm{~cm}^{-1}$ cation band lies $10-20 \mathrm{~cm}^{-1}$ beyond the upper limit to the frequency range normally attributed to nonadjacent hydrogens. Nevertheless, we attribute this to an out-of-plane bend of the nonadjacent hydrogens in the pentacene cation both because it is about 100 $\mathrm{cm}^{-1}$ below the lowest frequencies normally attributed to a $\mathrm{CH}$ in-plane bend and because its position, integrated intensity, and 
frequency shift from the corresponding neutral band $\left(\sim 30 \mathrm{~cm}^{-1}\right.$ to higher frequency) are all consistent with those of the nonadjacent $\mathrm{CH}$ out-of-plane bends in the other polyacene cations.

If the 933.9 and $748.5 \mathrm{~cm}^{-1}$ bands correspond respectively to the 900 and $731 \mathrm{~cm}^{-1} \mathrm{CH}$ out-of-plane bending features of neutral pentacene, we estimate (on the basis of the measured upper limit ionization efficiency of $12 \%$ ) that their intensities are suppressed relative to their value for the neutral molecule by a factor of 3.5. a value entirely consistent with those of the other polyacenes. On the other hand, the behavior of the 862.0 $\mathrm{cm}^{-1}$ band is anomalous. If the $862.0 \mathrm{~cm}^{-1}$ cation band corresponds to the $824 \mathrm{~cm}^{-1}$ neutral band, we find that its intensity is suppressed by a factor of 20 . While suppression by this magnitude is not unprecedented for a $\mathrm{CH}$ out-of-plane mode (the $\mathrm{CH}$ out-of-plane bend in the naphthalene cation is suppressed by a factor of 18 ), it is certainly one of the most extreme cases observed to date and conflicts with the much more modest value (3.5) observed for the other out-of-plane $\mathrm{CH}$ bends in the molecule.

Again, if these assignments to $\mathrm{CH}$ out-of-plane bends are correct, and important cation bands have not been missed due to screening by neutral species, the force field for the cation is sufficiently different from that of the neutral that these out-ofplane motions are not only reduced dramatically in intensity, but that the bond strengths and interactions are altered as well.

The Pentacene Anion, $\mathrm{C}_{22} \mathrm{H}_{14}{ }^{+}$. The photolytic behavior of matrix-isolated pentacene is unique from that of other PAHs investigated previously in one very important respect. The spectra of photolyzed Ar/pentacene samples reproducibly display a family of 15 additional bands whose strongest features appear with an intensity equal to or even surpassing that of the strongest cation features. The most prominent members of this family, with relative intensities listed in parentheses, fall at 712.7 (0.0439), $844.8(0.0668), 1158.8(0.196), 1218.8(0.207), 1349.2$ $(0.679), 1355.5(0.153), 1372.8(1.00), 1524.3(0.0275), 1539.6$ $(0.0903)$, and $1549.5(0.115) \mathrm{cm}^{-1}$. Most of these bands are shown in Figure 6. However, just as reproducibly, the spectra of photolyzed $\mathrm{Ar} / \mathrm{CCl}_{4} /$ pentacene samples fail to show any trace of these features despite the enhancement that is observed in the cation bands. Furthermore, it is perhaps suggestive that a number of these features seem paired with cation features (e.g., $1218.8,1349.2$, and $1372.8 \mathrm{~cm}^{-1}$ ), appearing consistently with similar intensity and $\sim 20 \mathrm{~cm}^{-1}$ lower in frequency. We believe that the observed behavior of these bands is consistent with their originating from the anion of pentacene. In experiments which do not involve an electron acceptor, the neutral pentacene molecules themselves accept the electrons generated by photolysis, forming the anion of the molecule. For those experiments which do employ an electron acceptor, $\mathrm{CCl}_{4}$ is present in the matrix at a much higher concentration than the PAH and presumably scavenges the free electrons preventing anion formation. The fact that pentacene has the highest electron affinity $(\sim 1-1.5 \mathrm{eV})$ of any of the PAHs which have been studied to date (typically EA $\$ 0.5 \mathrm{eV}$ ) is also consistent with this scenario. If this interpretation is indeed correct, it indicates that there is a distinct electron affinity threshold between $\sim 0.5$ and $1.0 \mathrm{eV}$ for the formation of matrix-isolated anions. Molecular species which fall below this limit may not be observable in their anionic form while isolated in matrices. Further investigation of this phenomenon will be necessary to fully understand the observations.

It should be noted that the production of matrix-isolated anions is by no means unprecedented. The application of electron spin resonance (ESR) spectrometry to the study of
TABLE 2: Near-Infrared Frequencies and Relative Intensities of the Polyacene Cations through Pentacene

\begin{tabular}{|c|c|c|c|c|c|c|c|}
\hline \multicolumn{2}{|c|}{ naphthalene } & \multicolumn{2}{|c|}{ anthracene } & \multicolumn{2}{|c|}{ tetracene } & \multicolumn{2}{|c|}{ pentacene } \\
\hline$v\left(\mathrm{~cm}^{-1}\right)$ & $I_{r \in l}$ & $\nu\left(\mathrm{cm}^{-1}\right)$ & $I_{\text {rel }}$ & $\overline{v\left(\mathrm{~cm}^{-1}\right)}$ & $I_{\text {rel }}$ & $\overline{v\left(\mathrm{~cm}^{-1}\right)}$ & $I_{\mathrm{rel}}$ \\
\hline 14805 & 1.00 & 13832 & 1.00 & $\begin{array}{l}11514 \\
11897 \\
12472 \\
12904 \\
13055 \\
13404\end{array}$ & $\begin{array}{l}1.00 \\
0.761 \\
0.130 \\
0.034 \\
0.064 \\
0.301\end{array}$ & $\begin{array}{c}8750.0 \\
10149 \\
10472 \\
10642 \\
10733 \\
11366 \\
11871 \\
12129\end{array}$ & $\begin{array}{l}0.018 \\
0.133 \\
1.00 \\
0.028 \\
0.042 \\
0.036 \\
0.374 \\
0.011\end{array}$ \\
\hline
\end{tabular}

matrix-isolated anions was pioneered more than 20 years ago ${ }^{21}$ and has been applied in many areas. ${ }^{22}$ The fact that those previous studies have involved species with electron affinities generally in the $2-4 \mathrm{eV}$ range and that the sensitivity of ESR spectrometry to radical anions far surpasses that of infrared spectroscopy does not conflict with the suggestion that the spectrum of the pentacene radical anion may be observed in our experiments.

B. Near-Infrared Spectroscopy. The band frequencies, areas, and relative intensities listed in Table 2 for the near-IR spectra were measured on the same matrices for which the midIR bands listed in Table 1 were measured. An interesting trend emerges in the ratio of the strongest near-infrared cation band to the strongest mid-infrared cation band as we move across the polyacene family from its smallest to its largest members. The ratio for the naphthalene cation has been reported previously as $\mathrm{F}\left(I(14805) / I(1218)=88 .^{10.11}\right.$ The ratio for the anthracene cation is slightly smaller, $(I(13832) / I(1341)=78$. For the larger members of the polyacene family, however, the ratio is dramatically reduced. For tetracene $(I(11514) / I(1358)=29$. and for pentacene it is only $(I(10472) / /(1396)=13$. We believe that this decrease is due primarily to the remarkable strength of the mid-infrared bands of tetracene and pentacene cations, rather than unusually small near-infrared, electronic band strengths. Despite having ion yield upper limits similar to those found with many other PAHs $(\sim 10 \%)$, the tetracene and pentacene cation bands are far and away the most intense features observed for any of the PAH cations which have been studied in our laboratory. In view of the importance of absolute integrated absorbance values, and the uncertainties in the PAH cation literature concerning absorbance values, we are developing an independant method to determine this quantity for PAH cations. The results of these experiments will be reported separately.

The near-infrared data are presented on an individual basis below.

The Naphthalene Cation, $\mathrm{C}_{10} \mathrm{H}_{8}{ }^{+}$. The near-infrared spectrum of the naphthalene cation isolated in an argon matrix is shown elsewhere. ${ }^{11,23,24}$ The frequency of the strongest band is listed in Table 2.

The Anthracene Cation, $\mathrm{C}_{14} \mathrm{H}_{10}{ }^{+}$. The near-infrared spectrum of the anthracene cation is given in ref 12 and 23 . The frequency of the strongest band is listed in Table 2. The nearinfrared spectrum of the anthracene cation isolated in an argon matrix has been discussed by Andrews et al. ${ }^{25}$ and Szczepanski et al. ${ }^{12}$

The Tetracene Cation, $\mathrm{C}_{18} \mathrm{H}_{12}{ }^{+}$. The near-infrared spectrum of the tetracene cation is shown in Figure 8 with frequencies and relative intensities listed in Table 2 . The near-infrared spectrum of the tetracene cation isolated in argon has been discussed by Andrews et al. ${ }^{25}$

The Pentacene Cation, $\mathrm{C}_{22} \mathrm{H}_{14}{ }^{+}$. The near-infrared spectrum of the pentacene cation is shown in Figure 9 with frequencies and relative intensities listed in Table 2. 


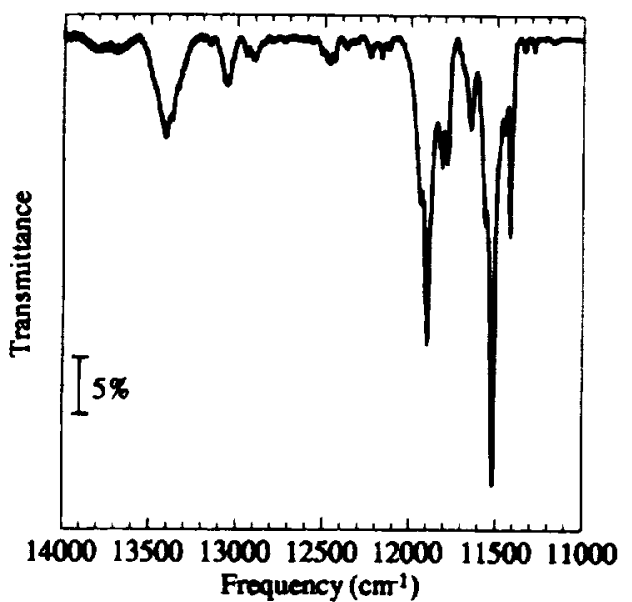

Figure 8. Near-infrared spectrum of the matrix isolated tetracene cation. This is of the same sample measured in the mid-infrared and shown in Figure 4 (argon matrix. $10 \mathrm{~K}$ )

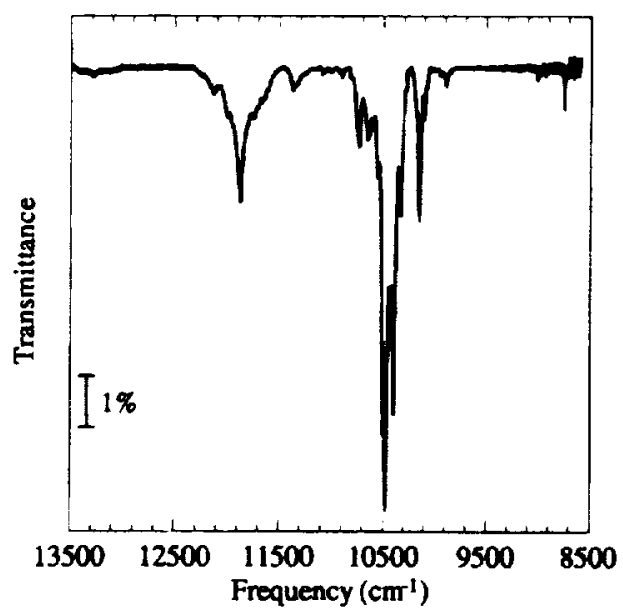

Figure 9. Near-infrared spectrum of the matrix isolated pentacene cation. This is of the same sample measured in the mid-infrared and shown in Figure 6 (argon matrix, $10 \mathrm{~K}$ ).

\section{Astrophysical Implications}

The general spectral properties of the polyacenes reported here are consistent with the results of previous studies on other PAH cations which showed the following: (i) The intensity of the bands in the $1600-1200 \mathrm{~cm}^{-1}$ range, the CC stretching and $\mathrm{CH}$ in-plane bending region of $\mathrm{PAH}$ cations, are typically 2-5 times greater than that of the $\mathrm{CH}$ out-of-plane bending modes which fall between 900 and $550 \mathrm{~cm}^{-1}$. (ii) The $\mathrm{CH}$ out-ofplane bending mode of the cations is typically 5-20 times weaker than the out-of-plane bending mode of the neutrals. (iii) The bands in the $\mathrm{CC}$ stretching and $\mathrm{CH}$ in-plane bending region are generally an order of magnitude stronger than that for the corresponding transitions in neutral PAHs. This behavior confirms that predicted theoretically by Defrees et al. ${ }^{15}$ and Pauzat et al. ${ }^{16}$ for PAH cations and supports the interstellar PAH model.

The most intense bands of all PAH cations studied to date fall within the envelopes of the most intense interstellar features, namely, those at 1610 and $1310 \mathrm{~cm}^{-1}(6.2$ and $7.7 \mu \mathrm{m})$. The observation that the most intense interstellar emission features fall in the $\mathrm{PAH} \mathrm{CC}$ stretching and $\mathrm{CH}$ in-plane-bending regions is completely consistent with the composite emission spectrum expected from a mixture dominated by free, ionized, small PAHs. The strongest absorptions in the polyacenes anthracene, tetracene, and pentacene fall between about 1500 and $1340 \mathrm{~cm}^{-1}$
$(6.7$ and $7.5 \mu \mathrm{m})$ and near $1180 \mathrm{~cm}^{-1}(8.5 \mu \mathrm{m})$, regions of only moderately intense interstellar emission between the strongest interstellar emission bands. These very strong polyacene bands also tend to fall in gaps in the spectra of the other PAH cations studied to date, suggesting that while PAHs with polyacene structures may contribute to portions of the interstellar emission spectra, they are not dominant members of the interstellar PAH family

\section{Conclusions}

The near- and mid-infrared spectra of the anthracene, tetracene, and pentacene cations isolated in argon matrices are presented. The mid-infrared spectrum of the argon matrix isolated pentacene anion is also reported. Ions were generated by in-situ photolysis.

The strongest mid-infrared absorption bands of these PAH cations fall between 1600 and about $1200 \mathrm{~cm}^{-1}$ and near 1180 $\mathrm{cm}^{-1}$, the $\mathrm{CC}$ stretching and $\mathrm{CH}$ in-plane bending region. These tend to be 2-5 times more intense than the bands due to the $\mathrm{CH}$ out-of-plane bending modes. The strongest bands tend to fall in groupings between 1500 and $1300 \mathrm{~cm}^{-1}$. On the other hand, the $\mathrm{CH}$ out-of plane bending modes in the cation are strongly depressed with respect to the neutrals. Only weak new bands grow in the normally very strong $\mathrm{CH}$ out-of-plane bending region between about 900 and $600 \mathrm{~cm}^{-1}$. The extent to which these out-of-plane motions are reduced in intensity and the bond strengths and interactions are altered is far more than would be expected on first principles by the removal of one electron from the $\pi$ system. Similarly, as has been the case with previous $\mathrm{PAH}$ cations studied, no new features were found in the $\mathrm{CH}$ stretch region between 3200 and $2900 \mathrm{~cm}^{-1}$, implying that the $\mathrm{CH}$ stretching modes of the cation are comparable to or weaker than for the neutral species. Theory predicts them to be weaker. ${ }^{15}$

The strongest cation bands coincide with $\mathrm{CC}$ stretching and $\mathrm{CH}$ in-plane bending modes, behavior just opposite that for the neutral species. This is important for the interpretation of the astronomical emission spectra which are dominated by very intense features in this region. Since most PAHs in the emission zones would be ionized, this apparent spectral characteristic of PAH cations gives strong support to the interstellar PAH hypothesis. The strongest absorptions in the polyacenes anthracene, tetracene, and pentacene tend to group around 1400 $\mathrm{cm}^{-1}$ (between about 1340 and $1500 \mathrm{~cm}^{-1}$ ) and near $1180 \mathrm{~cm}^{-1}$, regions of only moderately intense interstellar emission between the strongest interstellar emission bands. These very strong polyacene bands also tend to fall in gaps in the spectra of the other PAH cations studied to date, suggesting that while PAHs with polyacene structures may contribute to portions of the interstellar emission spectra, they are not dominant members of the interstellar PAH family

Acknowledgment. The authors acknowledge the expert technical support of Bob Walker and valuable scientific discussions with Farid Salama. Scott Sandford, and Christine Joblin. This work was fully supported by NASA's Laboratory Astrophysics and Long Term Space Astrophysics programs, under Grants 188-44-57-01 and 399-20-01-05.

Note Added in Proof. Recent unpublished calculations by Langhoff on the mid-infrared spectra of PAH cations and anions show good agreement with the experimental frequencies and most relative intensities reported here. However, the intensity ratio for the strongest bands in the pentacene cation are reversed in the calculations. 


\section{References and Notes}

(1) Allamandola. L. J.; Tielens. A. G. G. M.: Barker. J. R. Astrophys. J. Suppl. Ser. 1989, 71, 733. (b) Allamandola, L. J. In Topics in Current Chemistry: Cyvin. S.. Gutman. J., Eds.; Springer-Verlag: Berlin 1990; p

1. (c) Puget, J. L.. Leger, A. Annu. Rev. Astron. Astrophys. 1989, 27, 161

(2) Harris. S. J.: Weiner, A. M. Combust. Sci. Technol. 1983, 31. 155

(b) Frenklach. M.: Warnatz. J. Combust. Sci. Technol. 1987, 51, 265.

(3) Harvey, R. G.. Ed. Polvcyclic Hydrocarbons and Carcinogenesis, American Chemical Society: Washington. DC. 1985.

(4) Allamandola. L. J.: Tielens. A. G. G. M.: Barker. J. R. Astrophys. J. Lett. 1985, 290. L25.

(5) Leger. A.; Puget, J. L. Astron. Astrophys. 1984, 137, L5.

(6) For example: (a) Cyvin. B. N.: Klaeboe. P.: Whitmer, J. C.; Cyvin. S. J. Z Naturforsch. 1982, 37A, 251. (b) Cyvin, S. J.: Cyvin, B. N.: Brunvoll. J.: Whitmer. J. C.: Klaeboe. P.: Gustavsen, J. E. Z. Naturforsch. 1979, 34A 876.

(7) Sadiler Atlas of Infrared Spectra: Sadtler Research Laboratories: Philadelphia, PA, 1966.

(8) Witteborn, F. C.; Sandford, S. A.; Bregman, J. D.; Allamandola. L. J.; Cohen, M.; Wooden, D. H.; Graps, A. L. Astrophys. J. 1989, 34 I. 270.

(9) Hudgins, D. M.: Sandford. S. A.; Allamandola. L. J. J. Phys. Chem. 1994. $98,4243$.

(10) Hudgins D. M.; Allamandola. L. J. J. Phys. Chem. 1995, 99, 3033.

(11) Szczepanski. J.: Roser. D.: Personette. W.; Eyring. M.: Pellow. R.; Vala, M. J. Phys. Chem. 1992. 96. 7876.

(12) Szczepanski. J.; Vala. M.: Talbi, D.: Parisel, O.; Ellinger. Y.; J. Chem. Phys. 1993, $98,4494$.
(13) Hudgins, D. M.: Sandford, S. A. J. Phys. Chem. manuscript in preparation.

(14) Hudgins, D. M.; Sandford, S. A. Astrophys. J.. manuseript in preparation.

(15) (a) DeFrees, D. J.; Miller. M. D. In Interstellar Dust: Contributed Papers; Allamandola. L. J.. Tielens, A. G. G. M.. Eds.: NASA CP 3036. 1989; p 173. (b) DeFrees, D. J.: Miller, M. D.: Talbi, D.; Pauzat, F.; Ellinger, Y. Astrophys. J. 1993,408,530.

(16) Pauzat, F.; Talbi. D.; Miller, M. D.: DeFrees. D. J.: Ellinger. Y. J. Phys. Chem. 1992. 96. 7882.

(I7) Vaia, M.; Szczepanski, J.: Pauzat, F.; Parisel. O,; Talbi, D.: Ellinger, Y. J. Phys Chem. in press.

(18) Szczepanski, J.; Chapo, C.: Vala. M. Chem. Phys. 1993, 205.434.

(b) Szczepanski. J. Vala. M. Astrophys. J. 1993, 414,179.

(19) d'Hendecourt, L. B.; Leger. A.. In The First Symposium on the Infrared Cirrus and Diffuse Interstellar Clouds, ASP Conference Series Vol. 58: Cutri. R. M., Latter, W. B.. Eds.; Astronomical Society of the Pacific: San Francisco, 1994.

(20) White. C. M. J. Chem. Eng. Data 1986, 31, 198

(21) Kasai. P. H. Acc. Chem. Res. 1971, 4, 329

(22) For example: (a) Kasai. P. H. Phys. Rev Lett. 1968. 21, 67. (b) Kasai, P. H.: McLeod. D., Jr. J. Chem.Phys. 1969. 51. 1250.

(23) Andrews. L.: Kelsell, B. J.: Blankenship. T. A. J. Phvs. Chem. 1982. 86. 2916.

(24) Salama, F.; Allamandola, L. J. J. Chem. Phys. 1991. 94, 6964

(25) Andrews. L.: Friedman, S.: Kelsall, J. J. Phys. Chem 1985, 89, 4016.

JP9427084 
